\title{
Soft Pomeron in holographic QCD
}

\author{
Alfonso Ballon-Bayona, ${ }^{1}$ Robert Carcassés Quevedo, ${ }^{1}$ Miguel S. Costa, ${ }^{1,2}$ and Marko Djurici ${ }^{1}$ \\ ${ }^{1}$ Centro de Física do Porto e Departamento de Física e Astronomia da Faculdade de Ciências da \\ Universidade do Porto, Rua do Campo Alegre 687, 4169-007 Porto, Portugal \\ ${ }^{2}$ Theory Division, Department of Physics, CERN, CH-1211 Genève 23, Switzerland
}

(Received 18 September 2015; published 4 February 2016)

\begin{abstract}
We study the graviton Regge trajectory in holographic QCD as a model for high energy scattering processes dominated by soft-Pomeron exchange. This is done by considering spin $J$ fields from the closed string sector that are dual to glueball states of even spin and parity. In particular, we construct a model that governs the analytic continuation of the spin $J$ field equation to the region of real $J<2$, which includes the scattering domain of the negative Maldelstam variable $t$. The model leads to approximately linear Regge trajectories and is compatible with the measured values of 1.08 for the intercept and $0.25 \mathrm{GeV}^{-2}$ for the slope of the soft Pomeron. The intercept of the secondary Pomeron trajectory is in the same region of the subleading trajectories, made of mesons, proposed by Donnachie and Landshoff, and should therefore be taken into account.
\end{abstract}

DOI: 10.1103/PhysRevD.93.035005

\section{INTRODUCTION}

The Pomeron plays a crucial role in QCD Regge kinematics, for processes dominated by exchange of the vacuum quantum numbers. This includes elastic scattering of soft states at high energies and low momentum transfer. The corresponding amplitude exhibits a universal behavior explained within Regge theory [1],

$$
\mathcal{A}(s, t) \approx \beta(t) s^{\alpha(t)}, \quad \alpha(t)=1.08+0.25 t,
$$

in $\mathrm{GeV}$ units and for some function $\beta(t)$ that depends on the scattered states. A precise computation of the values of the intercept $\left(\alpha_{0}=1.08\right)$ and slope $\left(\alpha^{\prime}=0.25 \mathrm{GeV}^{-2}\right)$ is beyond our current understanding of QCD, since longrange strong interaction effects are important.

The gauge-gravity duality is a new tool to unveil QCD strongly coupled physics [2]. In particular, the Pomeron is conjectured to be the graviton Regge trajectory of the dual string theory [3]. This fact has been explored in diffractive processes dominated by Pomeron exchange, like low- $x$ deep inelastic scattering (DIS) [4-6], deeply virtual Compton scattering [7], vector meson production [8] and double diffractive Higgs production [9].

Consider for instance the case of low- $x$ DIS. One observes a rise of the intercept $j_{0}$ from 1.1 to 1.4 as $Q$ grows, where $\mathrm{Q}$ is the momentum scale of the photon probe. The conventional approach is to start from the perturbative Balitsky-Fadin-Kuraev-Lipatov (BFKL) hard Pomeron [10], which still exhibits conformal symmetry.

Published by the American Physical Society under the terms of the Creative Commons Attribution 3.0 License. Further distribution of this work must maintain attribution to the author(s) and the published article's title, journal citation, and DOI.
Introducing a cutoff, one explains the observed rise of the structure functions and even the running of the intercept, provided the cut $Q^{2}>4 \mathrm{GeV}^{2}$ is imposed in the kinematics [11]. However, dual models that also start from a conformal limit and introduce a hard wall cutoff in anti-de Sitter (AdS) space give even better fits to data, without imposing any restriction in the kinematics [6]. This is a strong motivation in favor of treating soft-Pomeron physics using the gauge/gravity duality.

This paper builds a soft-Pomeron phenomenology in holographic QCD. More concretely, we show that the Regge theory for spin $J$ exchanges in the dual geometry leads to the behavior (1) for the amplitude between soft probes.

\section{HOLOGRAPHIC QCD MODEL}

We will consider the holographic QCD model proposed in the works [12-14] based on gravity plus a dilaton field. We shall be working in the string frame because the Regge trajectory we are interested in is made of fundamental closed string states. As usual, the scalar field $\Phi=\Phi(z)$ and the dual geometry has the metric

$$
d s^{2}=g_{a b} d x^{a} d x^{b}=e^{2 A(z)}\left(d z^{2}+\eta_{\alpha \beta} d x^{\alpha} d x^{\beta}\right),
$$

where $\eta_{\alpha \beta}$ is the Minkowski boundary metric. In the string frame the corresponding action is

$$
S=\frac{1}{2 \kappa^{2}} \int d^{5} x \sqrt{-g} e^{-2 \Phi}\left[R+4(\partial \Phi)^{2}+V\right],
$$

with 


$$
V=e^{-\frac{4}{3} \Phi}\left[\frac{64}{27} W^{2}-\frac{4}{3}\left(\frac{d W}{d \Phi}\right)^{2}\right] .
$$

The field $\Phi$ is the dilaton without the zero mode that is absorbed in the gravitational coupling $\kappa$. The field equations arising from (3) take the form

$$
\begin{aligned}
R_{a b}+2 \nabla_{a} \nabla_{b} \Phi-\frac{1}{4} \frac{d V}{d \Phi} & g_{a b}=0, \\
2 \nabla^{2} \Phi-4(\nabla \Phi)^{2}+V+\frac{3}{4} \frac{d V}{d \Phi} & =0 .
\end{aligned}
$$

The superpotential $W(\Phi)$ is fixed phenomenologically by demanding that the model reproduces basic QCD data, such as the beta function, heavy quark/antiquark linear potential and glueball spectrum. In this work we take the Background I of $[12,13]$ where

$$
W=\frac{9}{4 L}\left(1+\frac{2}{3} b_{0} \lambda\right)^{\frac{2}{3}}\left[1+\frac{\left(2 b_{0}^{2}+3 b_{1}\right) \log \left(1+\lambda^{2}\right)}{18 a}\right]^{\frac{4 a}{3}},
$$

$\lambda=e^{\Phi}$ and the length scale $L$ fixes the units.

The 't Hooft coupling of the dual Yang-Mills theory $\bar{\lambda}$ is fixed by $\lambda$ up to a multiplicative constant, i.e. $\bar{\lambda}=c_{0} \lambda$. For the model considered in this work the constants in (6) are given by

$$
b_{0}=4.2, \quad \frac{b_{1}}{b_{0}^{2}}=\frac{51}{121}, \quad a=\frac{3}{16} .
$$

The model has an additional integration constant that can be related to $\Lambda_{\mathrm{QCD}}$, via the identification of the energy scale and warp factor, $\log E=A(z)-\frac{2}{3} \Phi(z)$. As shown in $[12,13]$, the UV behavior of the superpotential (6) leads to a beta function

$$
\beta=\frac{d \lambda}{d \log E}=-b_{0} \lambda^{2}-b_{1} \lambda^{3}+\cdots .
$$

This is consistent with the two-loop perturbative beta function in large- $N$ Yang-Mills

$$
\bar{\beta}=-\bar{b}_{0} \bar{\lambda}^{2}-\bar{b}_{1} \bar{\lambda}^{3}, \quad \bar{b}_{0}=\frac{2}{3} \frac{11}{(4 \pi)^{2}}, \quad \frac{\bar{b}_{1}}{\bar{b}_{0}^{2}}=\frac{51}{121},
$$

if we take $c_{0}=b_{0} / \bar{b}_{0}$. This fixes the second parameter in (7). The other parameters are fixed by the IR constraints coming from confinement and an asymptotic linear glueball spectrum and lattice QCD.

Given that all of the parameters are already fixed at this point, one may ask how the field theory coupling runs with energy. Setting $N_{c}=3$ the QCD running coupling can be identified with $\alpha_{s}=\bar{\lambda} /(12 \pi)$. Figure 1 shows how $\alpha_{s}$ runs

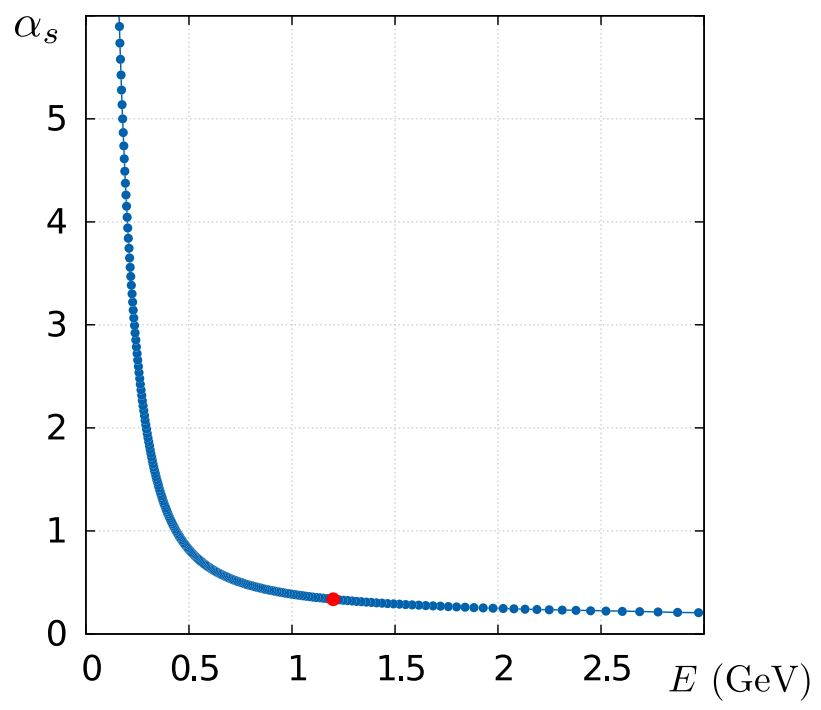

FIG. 1. Running coupling $\alpha_{s}$ vs energy scale. The red point is $\alpha_{s}(1.2 \mathrm{GeV})=0.34$.

with the energy scale in the model, giving 0.34 for the value for $E=1.2 \mathrm{GeV}$, which is very close to the experimental value 0.35 .

We can recover the conformal limit by considering the parameters $b_{0}=b_{1}=0$. Then we can set $\Phi=0$, the superpotential becomes the cosmological constant $-12 / L^{2}$, and the metric becomes that of AdS space, i.e. $A(z)=\ln (L / z)$.

\section{POMERON IN HOLOGRAPHIC QCD}

\section{A. Graviton}

Since we are interested in the graviton Regge trajectory let us start by considering perturbations to the background in the string frame. We shall write the metric and the dilaton, respectively, as

$$
g_{a b}+h_{a b}, \quad \Phi+\varphi .
$$

It is then a mechanical computation to obtain from (5) the linearized equations of motion for the perturbations $h_{a b}$ and $\varphi$,

$$
\begin{aligned}
& \nabla^{2} h_{a b}-2 \nabla_{(a} \nabla^{c} h_{b) c}+\nabla_{a} \nabla_{b} h+2 R_{a c b d} h^{c d} \\
& +4 \nabla^{c} \nabla_{(a} \Phi h_{b) c}+2 \nabla^{c} \Phi\left(2 \nabla_{(a} h_{b) c}-\nabla_{c} h_{a b}\right) \\
& -4 \nabla_{a} \nabla_{b} \varphi+\frac{1}{2} g_{a b} V^{\prime \prime}(\Phi) \varphi=0, \\
& \nabla^{2} \varphi+\frac{1}{2} V^{\prime}(\Phi) \varphi+\frac{3}{8} V^{\prime \prime}(\Phi) \varphi \\
& -4 \nabla \varphi \cdot \nabla \Phi-\frac{1}{2} \nabla^{a} \Phi\left(2 \nabla^{b} h_{a b}-\nabla_{a} h\right) \\
& -h^{a b} \nabla_{a} \nabla_{b} \Phi+2 h^{a b} \nabla_{a} \Phi \nabla_{b} \Phi=0,
\end{aligned}
$$


where the covariant derivatives and the Riemann tensor refer to the background and $h=h_{a}{ }^{a}$. Field perturbations will be classified according to the $S O(1,3)$ global symmetry of the background. Thus we shall decompose the metric perturbations $h_{a b}$ as

$h_{\alpha \beta}=h_{\alpha \beta}^{T T}+\partial_{(\alpha} h_{\beta)}^{T}+\left(4 \partial_{\alpha} \partial_{\beta}-\eta_{\alpha \beta} \partial^{2}\right) \bar{h}+\eta_{\alpha \beta} h, \quad h_{z z}$,

$h_{z \alpha}=v_{\alpha}^{T}+\partial_{\alpha} s$.

As usual transverse and traceless (TT) tensor fluctuations, transverse vector fluctuations and scalar fluctuations decouple. Moreover, since we are interested only in the TT metric fluctuations, we do not need to worry about the mixing of perturbations. It is then simple to see that (11) gives for the TT metric fluctuations

$$
\left(\nabla^{2}-2 e^{-2 A(z)} \dot{\Phi} \nabla_{z}+2 \dot{A}^{2} e^{-2 A(z)}\right) h_{\alpha \beta}^{T T}=0 .
$$

The term with the dilaton arises from the usual coupling $-2 \partial^{c} \Phi \nabla_{c} h_{a b}$ for metric fluctuations in the string frame; the other term comes from the coupling to the Riemann tensor $R_{a c b d} h^{c d}$, with $R_{\alpha \mu \beta \nu}=\dot{A}^{2} e^{2 A}\left(\eta_{\alpha \nu} \eta_{\mu \beta}-\eta_{\alpha \beta} \eta_{\mu \nu}\right)$ and $R_{\alpha z \beta z}=-\ddot{A} e^{2 A} \eta_{\alpha \beta}$. In the case of pure AdS space, $A(z)=\ln (L / z)$, so (13) simplifies to

$$
\left(\nabla^{2}-m^{2}\right) h_{\alpha \beta}^{T T}=0,
$$

with $(L m)^{2}=-2$, as expected for the AdS graviton.

\section{B. Dual spin $J$ field}

We shall consider the exchange of twist 2 operators of Lorentz spin $J$ formed from the gluon field [15]

$$
\mathcal{O}_{J} \sim \operatorname{tr}\left[F_{\beta \alpha_{1}} D_{\alpha_{2}} \cdots D_{\alpha_{J-1}} F_{\alpha_{J}}^{\beta}\right],
$$

where $D$ is the QCD covariant derivative. The dimension of the operator $\mathcal{O}_{J}$ can be written as $\Delta=2+J+\gamma_{J}$, where $\gamma_{J}$ is the anomalous dimension. In free theory the operator has critical dimension $\Delta=2+J$.

Knowledge of the curve $\Delta=\Delta(J)$ is important when summing over spin $J$ exchanges, since this sum is done by analytic continuation in the $J$ plane, and then by considering the region of real $J<2$. Figure 2 summarizes a few important facts about the curve $\Delta=\Delta(J)$. Let us define the variable $\nu$ by $\Delta=2+i \nu$, and consider the inverse function $J=J(\nu)$. The figure shows the perturbative BFKL result for $J(\nu)$, which is an even function of $\nu$ and has poles at $i \nu=1$. Beyond perturbation theory, the curve must pass through the energy-momentum tensor protected point at $J=2$ and $\Delta=4$. We shall use a quadratic approximation to this curve that passes through this protected point,

$$
J(\nu) \approx J_{0}-\mathcal{D} \nu^{2}, \quad 4 \mathcal{D}=2-J_{0} .
$$

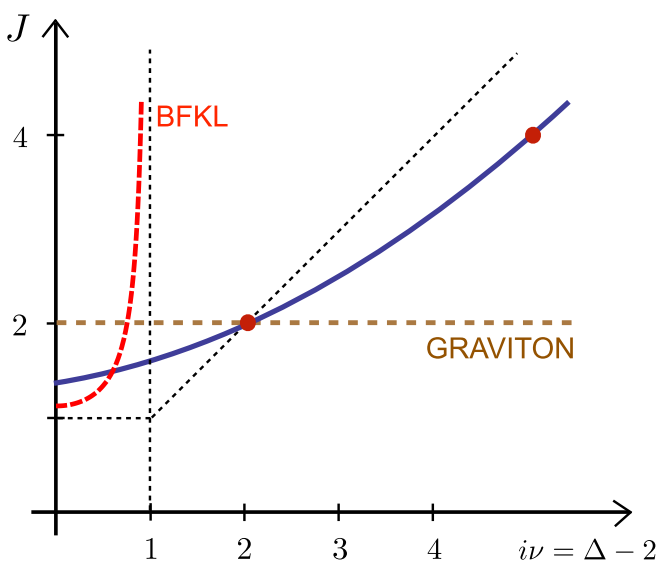

FIG. 2. Expected form of the $\Delta=\Delta(J)$ curve (in blue).

The use of a quadratic form for the function $J(\nu)$ is known as the diffusion limit and it is used both in BFKL physics and in dual models that consider the AdS graviton Regge trajectory (see for instance [7]).

Consider now the spin $J$ field dual to the twist 2 operators (15). For pure AdS this field obeys the equation

$$
\left(\nabla^{2}-m^{2}\right) h_{a_{1} \ldots a_{J}}=0, \quad(L m)^{2}=\Delta(\Delta-4)-J,
$$

where $L$ is the AdS length scale. Note that this field is symmetric, traceless and transverse $\left(\nabla^{b} h_{b a_{2} \ldots a_{J}}=0\right)$.

To consider the spin $J$ field in a general background of the form (2), we need again to do a decomposition in $S O(1,3)$ irreducible representations. The propagating degrees are described by components $h_{\alpha_{1} \cdots \alpha_{J}}$, since the other components $h_{z \cdots z \alpha_{i} \cdots \alpha_{J}}(i \geq 2)$ are fixed by the transversality condition. Thus we need to define the equation of motion for $h_{\alpha_{1} \ldots \alpha_{J}}$. Of course we do not know its form for the dual of QCD, but follow a phenomenological approach. We shall require that such equation is compatible with the spin 2 case (13), since in that case it must reduce to that of the graviton, whose dual operator has a protected dimension. Moreover, we require the coupling to the dilaton to be that of closed strings in the graviton Regge trajectory arising from the term $-2 \partial^{c} \Phi \nabla_{c} h_{a_{1} \cdots a_{J}}$. Finally, we require the equation to reduce to (17) in the conformal limit (constant dilaton). This leads to the following proposal:

$$
\left(\nabla^{2}-2 e^{-2 A} \dot{\Phi} \nabla_{z}-\frac{\Delta(\Delta-4)}{L^{2}}+J \dot{A}^{2} e^{-2 A}\right) h_{\alpha_{1} \ldots \alpha_{J}}=0,
$$

where here $L$ is a length scale parameter. It is trivial to verify that setting $J=2$ (and $\Delta=4$ ) this equation reduces to the the graviton equation (13). Similarly, setting $A(z)=$ $\ln (L / z)$ and $\Phi=0$ we recover the spin $J$ AdS equation (17). The dilaton term arises from considering tree 
level closed strings, which is justified since we work at large $N$. We expect that there will be more terms in this equation arising from other curvature couplings and derivatives of the dilaton field. Assuming the equation is analytic in $J$, these terms should be proportional to $J-2$, so that they are absent for $J=2$. Notice that there can be such terms still at the level of two derivatives, that is, terms proportional to

$$
e^{-2 A}\left(\dot{A}^{2}-\ddot{A}\right), \quad e^{-2 A} \dot{\Phi}^{2}, \quad e^{-2 A} \ddot{\Phi},
$$

which also vanish in the conformal limit. Terms with higher derivatives will appear in an $\alpha^{\prime} / L^{2}$ expansion. As already stated, we shall follow a phenomenological approach and use the simple form (18) to describe the fluctuations of the spin $J$ field in holographic QCD.

We will be interested in the continuation of (18) to the unphysical region of $J<2$. It is here that we will use the diffusion limit (16), writing in (18)

$$
\frac{\Delta(\Delta-4)}{L^{2}} \approx \frac{2}{l_{s}^{2}}(J-2),
$$

with $l_{s}$ a length scale set by the QCD string. Notice that we are fixing $l_{s}$ to a constant determined by IR physics, but in fact it should depend on energy scale, since the curve $\Delta=$ $\Delta(J)$ in Fig. 2 should vary with energy scale, keeping its general shape. However, for the soft Pomeron this should not matter [16]. We leave $l_{s}$ as a phenomenological parameter to be fixed by data.

In the Regge limit we are actually interested in the $+\cdots+$ component of (18). To find the solution write

$$
h_{+\cdots+}(z, x)=e^{i q \cdot x} e^{\frac{2 J-3}{2} A(z)+\Phi(z)} \psi(z),
$$

where $q \cdot x=\eta_{\alpha \beta} q^{\alpha} x^{\beta}$. Then, a computation shows that (18) reduces to the Schrödinger problem

$$
\begin{gathered}
\left(-\frac{d^{2}}{d z^{2}}+U(z)\right) \psi(z)=t \psi(z), \\
U(z)=\frac{15}{4} \dot{A}^{2}-5 \dot{A} \dot{\Phi}+\dot{\Phi}^{2}+\frac{\Delta(\Delta-4)}{L^{2}} e^{2 A(z)},
\end{gathered}
$$

with $t=-q^{2}$. The energy spectrum for each $J$ quantizes $t=t_{n}(J)$, therefore yielding the glueball masses.

\section{C. $t$-channel spin $\mathbf{J}$ exchange}

Next consider the elastic scattering of QCD hadronic states of masses $m_{1}$ and $m_{2}$. We write the incoming momenta $k_{1}, k_{2}$ and the outgoing momenta $k_{3}, k_{4}$ in light-cone coordinates $(+,-, \perp)$ as

$$
\begin{array}{ll}
k_{1}=\left(\sqrt{s}, \frac{m_{1}^{2}}{\sqrt{s}}, 0\right), & k_{3}=-\left(\sqrt{s}, \frac{m_{1}^{2}+q_{\perp}^{2}}{\sqrt{s}}, q_{\perp}\right), \\
k_{2}=\left(\frac{m_{2}^{2}}{\sqrt{s}}, \sqrt{s}, 0\right), & k_{4}=-\left(\frac{m_{2}^{2}+q_{\perp}^{2}}{\sqrt{s}}, \sqrt{s},-q_{\perp}\right),
\end{array}
$$

where we consider the Regge limit $s \gg t=-q_{\perp}^{2}$.

Each hadron is described by a normalizable mode $\Upsilon_{i}(z, x)=e^{i k_{i} \cdot x_{i}} v_{i}(z)$ where $v_{3}=v_{1}^{*}$ and $v_{4}=v_{2}^{*}$. The hadrons we consider are made of open strings. Then the coupling of each hadronic field to the spin $J$ closed string fields has the form

$$
\kappa_{J} \int d^{5} x \sqrt{-g} e^{-\Phi} h_{a_{1} \ldots a_{J}} \Upsilon \nabla^{a_{1}} \ldots \nabla^{a_{J}} \Upsilon
$$

Notice that in principle different types of hadrons will have a different coupling $\kappa_{J}$. The transverse condition on the spin $J$ field guarantees that this coupling is unique up to derivatives of the dilaton field, which are subleading in the Regge limit.

The amplitude for $m_{1} m_{2} \rightarrow m_{1} m_{2}$ scattering through exchange of a spin $J$ field in the $t$-channel may now be computed in the dual theory. In the Regge limit we have

$$
\begin{aligned}
\mathcal{A}_{J}\left(k_{i}\right)= & -\kappa_{J} \kappa_{J}^{\prime} \int d^{5} X d^{5} X^{\prime} \sqrt{-g} \sqrt{-g^{\prime}} e^{-\Phi-\Phi^{\prime}} \\
& \times\left(\Upsilon_{1} \partial_{-}^{J} \Upsilon_{3}\right) \Pi^{-\cdots-,+\cdots+}\left(X, X^{\prime}\right)\left(\Upsilon_{2}^{\prime} \partial_{+}^{\prime} \Upsilon_{4}^{\prime}\right),
\end{aligned}
$$

where $X=(z, x)$ and $X^{\prime}=\left(z^{\prime}, x^{\prime}\right)$ are bulk points and fields with a prime are evaluated at $X^{\prime}$, e.g. $\Phi^{\prime} \equiv \Phi\left(z^{\prime}\right)$. We use this notation throughout. We expect the spin $J$ field propagator to obey an equation of the type

$$
\begin{aligned}
(\mathcal{D} \Pi)_{a_{1} \ldots a_{J}, b_{1} \ldots b_{J}}\left(X, X^{\prime}\right)= & i e^{2 \Phi} g_{a_{1}\left(b_{1} \ldots g_{\left.\left|a_{J}\right| b_{J}\right)}\right.} \delta_{5}\left(X, X^{\prime}\right) \\
& - \text { traces, }
\end{aligned}
$$

for some differential operator $\mathcal{D}$. We are interested in the $+\cdots+,-\cdots-$ component of this equation, for which the differential operator $\mathcal{D}$ can be read from (18).

Some algebra shows the amplitude (26) simplifies to

$$
\begin{aligned}
\mathcal{A}_{J}(s, t)= & i V \frac{\kappa_{J} \kappa_{J}^{\prime}}{(-2)^{J}} s \int d z d z^{\prime} e^{3 A+3 A^{\prime}-\Phi-\Phi^{\prime}} \\
& \times\left|v_{1}\right|^{2}\left|v_{2}^{\prime}\right|^{2}\left(s e^{-A-A^{\prime}}\right)^{J-1} G_{J}\left(z, z^{\prime}, t\right),
\end{aligned}
$$

where $V$ is the boundary volume. The function

$$
G_{J}\left(z, z^{\prime}, t\right)=\int d^{2} l_{\perp} e^{-i q_{\perp} \cdot l_{\perp}} G_{J}\left(z, z^{\prime}, l_{\perp}\right),
$$

is the Fourier transform of 


$$
\begin{aligned}
G_{J}\left(z, z^{\prime}, l_{\perp}\right)= & i(-2)^{J} e^{(1-J)\left(A+A^{\prime}\right)} \\
& \times \frac{1}{2} \int d w^{+} d w^{-} \Pi_{+\cdots+,-\cdots-}\left(z, z^{\prime}, w\right),
\end{aligned}
$$

where $w=x-x^{\prime}=\left(w^{+}, w^{-}, l_{\perp}\right)$ and $l_{\perp}=x_{\perp}-x_{\perp}^{\prime}$. From the $+\cdots+,-\cdots-$ component of (27), as defined by (18), it follows that $G_{J}\left(z, z^{\prime}, l_{\perp}\right)$ is a Euclidean scalar propagator in the three-dimensional transverse space of the dual scattering process $\left[d x^{+}=d x^{-}=0\right.$ in (2)], i.e.

$$
\begin{aligned}
& {\left[\square_{3}-2 \dot{\Phi} \partial_{z}-e^{-2 A(z)}\left(2 \dot{A}^{2}+\ddot{A}-2 \dot{A} \dot{\Phi}\right)-\frac{\Delta(\Delta-4)}{L}\right]} \\
& \quad \times G_{J}\left(z, z^{\prime}, l_{\perp}\right)=-e^{2 \Phi} \delta_{3}\left(x, x^{\prime}\right)
\end{aligned}
$$

where here $x=\left(z, x_{\perp}\right)$ and $x^{\prime}=\left(z^{\prime}, x_{\perp}^{\prime}\right)$. Writing

$$
G_{J}\left(z, z^{\prime}, t\right)=e^{\Phi(z)-\frac{A(z)}{2}} \psi(z),
$$

the homogeneous solution to (31) is exactly given by the Schrödinger problem of (22) and (23). Moreover, using $\sum_{n} \psi_{n}(z) \psi_{n}^{*}\left(z^{\prime}\right)=\delta\left(z-z^{\prime}\right)$, we conclude that

$$
G_{J}\left(z, z^{\prime}, t\right)=e^{\Phi-\frac{A}{2}+\Phi^{\prime}-\frac{A^{\prime}}{2}} \sum_{n} \frac{\psi_{n}(z) \psi_{n}^{*}\left(z^{\prime}\right)}{t_{n}(J)-t}
$$

Note the eigenvalues $t_{n}$ and functions $\psi_{n}$ depend on $J$.

\section{Regge theory}

We will sum all even spin $J$ exchanges with $J \geq 2$ using a Sommerfeld-Watson transform,

$$
\frac{1}{2} \sum_{J \geq 2}\left(s^{J}+(-s)^{J}\right) \rightarrow-\frac{\pi}{2} \int \frac{d J}{2 \pi i} \frac{s^{J}+(-s)^{J}}{\sin (\pi J)}
$$

which requires the analytic continuation of the amplitude $\mathcal{A}_{J}(s, t)$ to the complex $J$ plane. Then, the amplitude for the exchange off all even spin $J$ fields becomes

$$
\mathcal{A}(s, t)=i V \int d z d z^{\prime} e^{3\left(A+A^{\prime}\right)}\left|v_{1}\right|^{2}\left|v_{2}^{\prime}\right|^{2} \sum_{n} \chi_{n},
$$

where $\chi_{n}=\chi_{n}\left(z, z^{\prime}, s, t\right)$ is given by

$$
\begin{aligned}
\chi_{n}= & -\frac{\pi}{2} \int \frac{d J}{2 \pi i} \frac{s^{J}+(-s)^{J}}{\sin (\pi J)} \frac{\kappa_{J} \kappa_{J}^{\prime}}{2^{J}} \\
& \times e^{-\left(J-\frac{1}{2}\right)\left(A+A^{\prime}\right)} \frac{\psi_{n}(z) \psi_{n}^{*}\left(z^{\prime}\right)}{t_{n}(J)-t} .
\end{aligned}
$$

We assume the $J$-plane integral can be deformed from the poles at even values of $J$ to the poles $J=j_{n}(t)$ defined by $t_{n}(J)=t$. In the scattering domain of negative $t$ these poles are along the real axis for $J<2$. Thus we can write

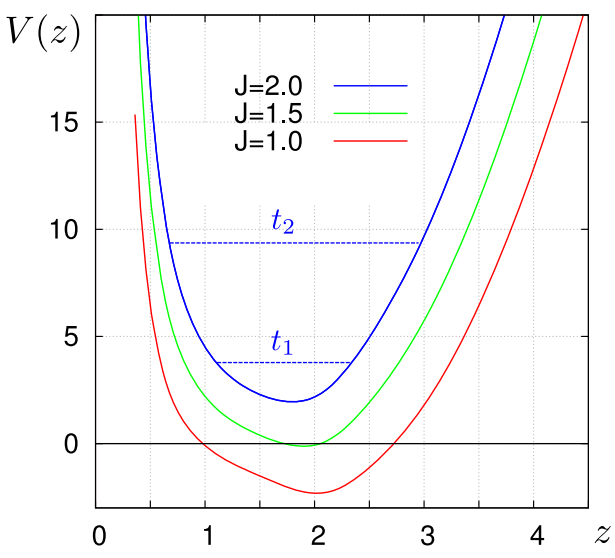

FIG. 3. Effective potential for different values of spin $J$. The first $2^{++}$glueball states are also shown.

$$
\begin{aligned}
\chi_{n}= & s^{j_{n}(t)}\left[-\frac{\pi}{2}\left(\cot \frac{\pi j_{n}}{2}+i\right) \frac{\kappa_{j_{n}} \kappa_{j_{n}}^{\prime}}{2^{j_{n}}}\right. \\
& \left.\times e^{-\left(j_{n}-\frac{1}{2}\right)\left(A+A^{\prime}\right)} \frac{d j_{n}}{d t} \psi_{n}(z) \psi_{n}^{*}\left(z^{\prime}\right)\right],
\end{aligned}
$$

where $j_{n}=j_{n}(t)$ and we remark that the wave functions $\psi_{n}$ are computed at $J=j_{n}(t)$. It is clear that for large $s$ the amplitude (35) will be dominated by the Regge pole with highest $j_{n}(t)$, in accord with the Regge behavior (1).

We now specify to the model considered in this paper, which is determined by the effective Schrödinger potential (23). Since we are interested in the region $J<2$, we can use the model introduced in (20) for the curve $\Delta=\Delta(J)$. Figure 3 shows the potential for several value of $J$. The energy levels for $J=2$ are shown and compute the mass of the spin 2 glueball masses. As $J$ decreases the energy levels will eventually cross the zero energy value. This will be the value of the intercept for the $n$th Reggeon. Figure 4 shows the curves $j_{n}(t)$, which clearly show that $n=1$ is the leading Regge pole. The curves are approximately straight so we can also define a Regge slope.

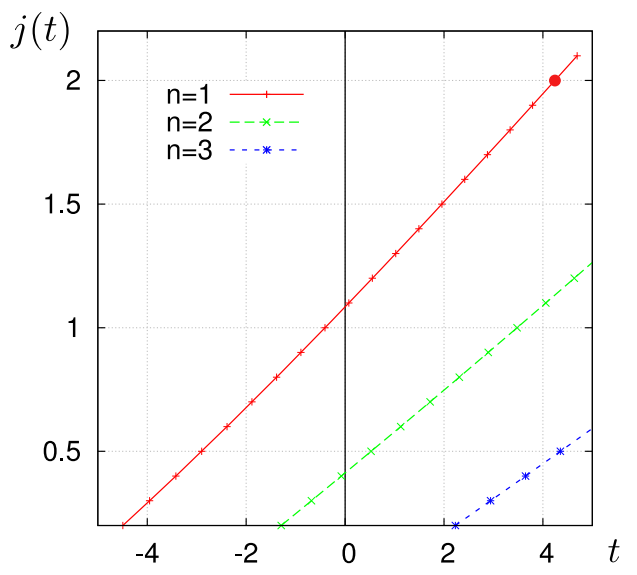

FIG. 4. The first Regge trajectories that result from solving the Schrödinger problem for discrete values of $J$. 


\section{RESULTS}

Finally we can test to which degree we are reproducing QCD physics. We consider first the leading Regge pole. We vary $l_{s}$, introduced in (20), to fix the Pomeron intercept to the value given in [1], as an optimal fit for total cross sections. For the value $l_{s}=0.178 \mathrm{GeV}^{-1}$, and independently of our choice of $\Lambda_{\mathrm{QCD}}$, we obtained $\alpha_{0}=1.08$. The value of the slope is then fixed by the choice of $\Lambda_{\mathrm{QCD}}$. We obtained $\alpha^{\prime} \Lambda_{\mathrm{QCD}}^{2}=0.018$. If we fix $\Lambda_{\mathrm{QCD}}=0.292 \mathrm{GeV}$ as in $[12,13]$, such that the first glueball mass $m_{0^{++}}=1.475 \mathrm{GeV}$, one obtains $\alpha^{\prime}=0.21 \mathrm{GeV}^{-2}$. If, on the other hand, we require the measured value of $\alpha^{\prime}=$ $0.25 \mathrm{GeV}^{-2}$ [17], we obtain $\Lambda_{\mathrm{QCD}}=0.265$. This is consistent with having the $2^{++}$glueball of the Pomeron trajectory with a mass of $1.9 \mathrm{GeV}$, which is a known possibility $[18,19]$.

Let us remark that we could fix $l_{s}$ to reproduce the intercept obtained in lattice simulations of $S U(3)$ pure Yang-Mills [20]. In this case, for $l_{s}=0.192 \mathrm{GeV}^{-1}$ one has $\alpha_{0}=0.93$. Then, setting $\Lambda=0.292$, which is fixed to reproduce $m_{0^{++}}=1.475 \mathrm{GeV}$ of the same lattice simulations, we obtained a slope $\alpha^{\prime}=0.25 \mathrm{GeV}^{-2}$. This is exactly the slope obtained by the lattice simulations [20].

For the second pole we obtained an intercept of 0.433 , which is consistent with the value used in [1]. We ran fits to $p \bar{p}$ total cross section data [21] and found that the second pole is necessary and needs to be in a narrow range of $\approx 0.35-0.55$. We determined this range by fitting an expression of the form

$$
\sigma=g_{0}\left(\alpha^{\prime} s\right)^{\alpha_{0}}+g_{1}\left(\alpha^{\prime} s\right)^{\alpha_{1}}
$$

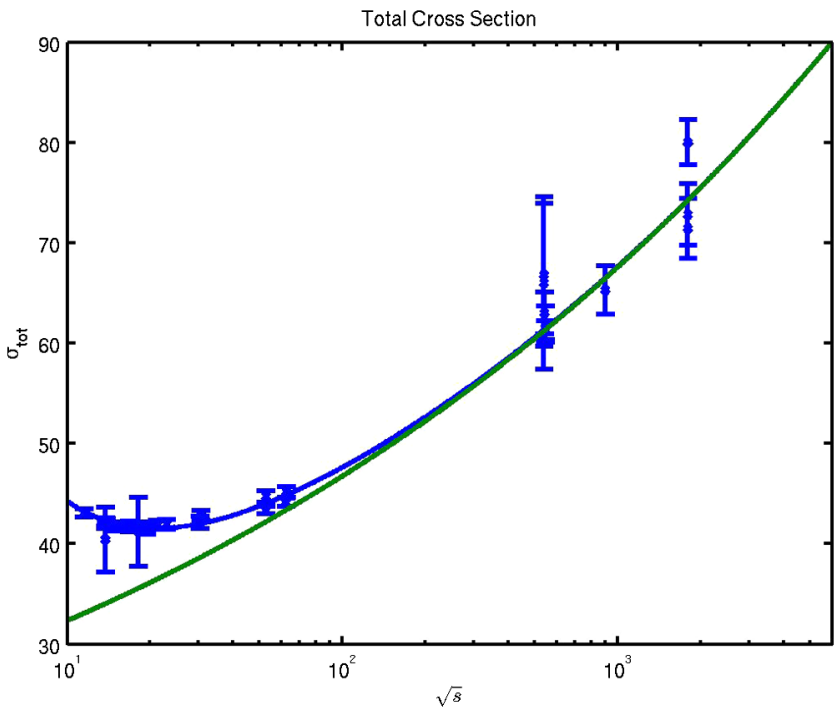

FIG. 5. A fit to $p \bar{p}$ total cross section data using the exchange of the first two Regge poles in our model. The green line represents the leading Pomeron exchange, and fails to fit the data at moderate values of $\sqrt{s}$. using $g_{0}$ and $g_{1}$ as parameters, and varying $\alpha_{1}$. We fit this to $p \bar{p}$ scattering data with $\sqrt{s}>10 \mathrm{GeV}$. The above range is fixed by the requirement that $\chi_{\mathrm{DOF}}^{2}$ be of order 1 or less. Our results can be seen in Fig. 5. As can be seen there using just the leading Pomeron exchange fails to fit the data satisfactorily. The second pole in [1] corresponds to several degenerate meson trajectories, while here it represents a next-to-leading glueball trajectory. Thus, our work points to the possibility that in this range there is a glueball trajectory as well. In fact, at least some of the $f_{2}$ states are known to correspond to glueballs (see [22] and references therein for recent results).

\section{CONCLUSION}

Soft-Pomeron physics is still beyond the current analytic understanding of QCD. The best one can do at weak coupling is to start from the BFKL approach and then introduce the running of the coupling, therefore breaking conformal symmetry. As a consequence, the branch cut of the BFKL Pomeron becomes a set of poles in the $J$ plane [11]. This approach can be used to fit DIS data for hard scattering, keeping a very large number of poles. However, it is not applicable to the case of soft probes. In general we expect to have a description of soft-Pomeron exchange as a Regge pole, in agreement with the phenomenological approach pioneered by Donnachie and Landshoff [1]. Such a description was proposed in [3], based on the scattering of closed strings in a dual confining background. In particular that work anticipated that for confining theories with a negative $\beta$ function the Pomeron, described as the graviton Regge trajectory, becomes a Regge pole. Our work confirms this expectation by extending the holographic QCD model of [14] to scattering processes dominated by soft-Pomeron exchange, bringing a new insight to soft-Pomeron physics.

Let us finish with a caveat and two open questions. It has been claimed that a soft-Pomeron pole is not enough to describe the new LHC data [23]. This is somewhat expected, since it is known that at very high energies such a Regge pole would violate the Froissart-Martin bound, and other effects need to be included, for example multipomeron exchange. However, this does not invalidate the great experimental successes of soft-Pomeron exchange up to LHC energies, as well as the necessity to understand the subleading trajectories.

The first question concerns the relation between hard and soft Pomerons. Recent studies in gauge/gravity duality reproduce a plethora of low- $x$ processes using the graviton Regge trajectory as the dual trajectory of the QCD Pomeron [4-9]. In these cases one observes a running of the intercept with the size of the probes. It would be very interesting if we could embed these results within the present model, therefore unifying both Pomerons. Another question is related to the spectrum of the spin $J$ field at integer values. It would be very nice to reconstruct the spin $J$ equation in this domain such that it reproduces perturbative QCD results. 


\section{ACKNOWLEDGMENTS}

We wish to thank Nick Evans, João Penedones, Chung-I Tan and Dimitrios Zoakos for the discussions. This research received funding from the (European Union) 7th Framework Programme (Marie Curie Actions) under Grant Agreements No. 269217 and No. 317089 (GATIS). The work of A. B. has been supported by the Brazilian agency CAPES, through the fellowship BEX 8051/14-3. A. B also thanks the Galileo Galilei Institute for Theoretical Physics for the hospitality and the INFN for partial support during the completion of this work. The work of M. D. has been supported by the Portuguese Fundação para a Ciencia e a Tecnologia (FCT) through the fellowship SFRH/BCC/ $105757 / 2014$.
[1] A. Donnachie and P. V. Landshoff, Total cross-sections, Phys. Lett. B 296, 227 (1992).

[2] J. M. Maldacena, The large $N$ limit of superconformal field theories and supergravity, Adv. Theor. Math. Phys. 2, 231 (1998).

[3] R. C. Brower, J. Polchinski, M. J. Strassler, and C. I. Tan, The Pomeron and gauge/string duality, J. High Energy Phys. 12 (2007) 005.

[4] L. Cornalba and M. S. Costa, Saturation in deep inelastic scattering from AdS/CFT, Phys. Rev. D 78, 096010 (2008).

[5] E. Levin and I. Potashnikova, Inelastic processes in DIS and $N=4$ SYM, J. High Energy Phys. 08 (2010) 112.

[6] R. C. Brower, M. Djuric, I. Sarcevic, and C. I. Tan, Stringgauge dual description of deep inelastic scattering at small$x$, J. High Energy Phys. 11 (2010) 051.

[7] M. S. Costa and M. Djuric, Deeply virtual compton scattering from gauge/gravity duality, Phys. Rev. D 86, 016009 (2012).

[8] M. S. Costa, M. Djuric, and N. Evans, Vector meson production at low $\mathrm{x}$ from gauge/gravity duality, J. High Energy Phys. 09 (2013) 084.

[9] R. C. Brower, M. Djuric, and C. I. Tan, Diffractive Higgs production by AdS Pomeron fusion, J. High Energy Phys. 09 (2012) 097.

[10] V. S. Fadin, E. A. Kuraev, and L. N. Lipatov, On the Pomeranchuk singularity in asymptotically free theories, Phys. Lett. B 60B, 50 (1975); E. A. Kuraev, L. N. Lipatov, and V.S. Fadin, The Pomeranchuk singularity in nonAbelian gauge theories, Sov. Phys. JETP 45, 199 (1977); Ya. Balitsky and L. N. Lipatov, The Pomeranchuk singularity in quantum chromodynamics, Sov. J. Nucl. Phys. 28, 822 (1978).

[11] H. Kowalski, L. N. Lipatov, D. A. Ross, and G. Watt, Using HERA data to determine the infrared behaviour of the BFKL amplitude, Eur. Phys. J. C 70, 983 (2010).
[12] U. Gursoy and E. Kiritsis, Exploring improved holographic theories for QCD: Part I, J. High Energy Phys. 02 (2008) 032.

[13] U. Gursoy, E. Kiritsis, and F. Nitti, Exploring improved holographic theories for QCD: Part II, J. High Energy Phys. 02 (2008) 019.

[14] U. Gursoy, E. Kiritsis, L. Mazzanti, G. Michalogiorgakis, and F. Nitti, Improved holographic QCD, Lect. Notes Phys. 828, 79 (2011).

[15] In the singlet sector there is also the twist 2 quark operator $\mathcal{O}_{J} \sim \bar{\psi} \Gamma_{\alpha_{1}} D_{\alpha_{2}} \ldots D_{\alpha_{J}} \psi$, however we are considering processes dominated by exchange of the gluon field.

[16] For instance, the approximation (20) misses the dimensions of the operators with $J>2$ in the free theory limit.

[17] G. A. Jaroszkiewicz and P. V. Landshoff, Model for diffraction excitation, Phys. Rev. D 10, 170 (1974).

[18] S. Donnachie et al., Pomeron Physics and QCD, 1st ed. (Cambridge University Press, Cambridge, England, 2002).

[19] F. Brünner, D. Parganlija, and A. Rebhan, Glueball decay rates in the Witten-Sakai-Sugimoto model, Phys. Rev. D 91, 106002 (2015).

[20] H. B. Meyer and M. J. Teper, Glueball Regge trajectories and the Pomeron: A lattice study, Phys. Lett. B 605, 344 (2005); H. B. Meyer, Glueball Regge trajectories, arXiv: hep-lat/0508002.

[21] K. A. Olive (Particle Data Group), Review of particle physics, Chin. Phys. C 38, 090001 (2014).

[22] J. Sonnenschein and D. Weissman, Glueballs as rotating folded closed strings, J. High Energy Phys. 12 (2015) 011.

[23] A. Donnachie and P. V. Landshoff, Elastic scattering at the LHC, arXiv:1112.2485; E. Gotsman, Amplitudes and cross sections at the LHC, arXiv:1304.7627. 